\title{
La escritura en las áreas curriculares
}

\author{
Writing in the curriculum areas
}

\author{
Silvia Valdivia Yábar ${ }^{1}$ y Juan López Chávez ${ }^{2}$
}

\section{RESUMEN}

El objetivo de esta comunicación es descubrir y escribir sobre lo que nosotros creemos que en última instancia importa en este terreno, la escritura por consiguiente, no se trata de elaborar una lista de actividades que motiven a los alumnos a escribir relatos, poesía o informes. No se trata de «poner» más escritura en las clases de las áreas curriculares. Los estudiantes ya pasan un considerable porcentaje de tiempo escribiendo. Pero de ese tiempo solo un mínimo porcentaje se invierte en escritura real; el resto está dedicado a llenar líneas de puntos. La escritura que se produce en las áreas curriculares puede dividirse en escritura mecánica (toma de notas, copia del pizarrón, respuestas cortas, etc.) y escritos de evaluación. En todas esas instancias, el propósito no es aprender sino mostrar a los docentes lo que el alumno sabe y no sabe.

Palabras clave: escritura; comunicación; áreas curriculares.

\section{ABSTRACT}

The purpose of this communication is to discover and write about what we believe ultimately matters in this field. Therefore, it is not about making a list of activities that encourage students to write stories, poetry or reports. It is not «put» more writing classes curricular areas. Students and spend considerable percentage of time writing. But at that time only a small percentage is invested in real handwriting; the rest is devoted to fill dotted lines. Writing that occurs in curricular areas can be divided into mechanical writing (taking notes, copying from the blackboard, short answer, etc.) and written evaluation. In all these instances, the purpose is not to learn but to show teachers what students know and do not know.

Keywords: writing; communication; curriculum areas.

\section{ICHIKLLACHAW}

Kay willakuychawqa musyanapaqwan qillqanapaqwanraykurmi rurakashqa, imanawmi nuqantsikpura kikintsikpita iñishintsik kay musyapakuychaw. Tsayqa manam ruraykuna hurqayllatsu, yachakuq wamrakunapa qillqaynin, harawin, willakuynin ayllupakuy rurayninllatsu manam. Tsayqa manam aryakunaman qillqaynin «churananllatsu». Yachakuq wamrakunaqa hunaq hunaq qillqayanqanpitanam shayaamun, tsaypita shamuykar rasunpaypa ichikllam kikinchaw qillqayninqa, wakin yachakuq wamrakunaqa

Consultora de UNICEF y del Ministerio de Educación del Perú.

2 Universidad Nacional Autónoma de México. 
chiku siqikunallata tinkutsinanllachawmi kakuyan. Kurikularkunapa aryankuna qillqayninqa ishkaymanmi rakikan: mikanika qillqayman (ichik willakuy qillqaykuna, qillqana pirqapita qillqaynin, uchuk yaskiykuna) hina chanintsay qillqaykunamanmi rakikan. Tsay llapanchaw manam yachakuyllatsu kanman, manatsayqa yachatsikuqkunatam rikaatsishwan yachakuq wamrakuna yachayanqantawan mana yachayanqantapis.

Pushaq shimikuna: qillqakuna; willanakuy; kurikular aryakuna.

\section{INTRODUCCIÓN}

Nos preguntamos qué pensamientos se pondrán en marcha cuando los lectores ven el título de este resumen. ¿Se imaginarán que encontrarán una lista de actividades sobre cómo utilizar la escritura en distintas áreas curriculares? Una lista de esas características no sería difícil de hacer. Presentar una serie de actividades es la parte sencilla de la escritura; sin embargo, decidir cuáles son pertinentes es algo más complicado. No es diferente de lo que le sucede a un pintor: él puede encontrar fácilmente azules, naranjas y verdes para poner en una tela; pero el desafío es saber dónde, cuándo y cómo incorporarlos. Lo difícil es tener una visión organizativa.

El objetivo en esta comunicación no es elaborar una lista de actividades que motiven a los alumnos a escribir relatos, poesía o informes, sino descubrir y escribir sobre lo que nosotros creemos que en última instancia importa en este terreno. Queremos ser claros en algo: no se trata de «poner» más escritura en las clases de las áreas curriculares. Los estudiantes ya pasan un considerable porcentaje de tiempo escribiendo. Pero de ese tiempo solo un mínimo porcentaje se invierte en escritura real; el resto está dedicado a llenar líneas de puntos. La escritura que se produce en las áreas curriculares puede dividirse en escritura mecánica (toma de notas, copia del pizarrón, respuestas cortas, etc.) y escritos de evaluación. En todas esas instancias, el propósito no es aprender sino mostrar a los docentes lo que el alumno sabe y no sabe.

Cuando se escribe para aprender, cuando se piensa lápiz en mano, cuando se hace preguntas, cuando se imagina hipótesis, cuando se organiza la información, el texto resultante no convencería a nadie de que se sabe. Esto vale para la mayor parte de la escritura para el currículum, actividades que ocupan un lugar muy reducido en nuestras aulas. Es importante mencionar que, al hacer esas operaciones, que serán descritas en esta comunicación, los aprendices adquieren una mayor conciencia y un mayor dominio de su pensamiento. Nosotros sostenemos que «la escritura nos da conciencia de nuestros pensamientos y nos permite organizarlos, nos obliga a detenernos sobre las ideas el tiempo suficiente para examinarlas, nos hace pensar sobre nuestros pensamientos». Releer la propia escritura, volver sobre las huellas del propio pensamiento, es indispensable.

\section{MATERIALES Y MÉTODOS}

Los datos que se analizan en el presente trabajo fueron recopilados mediante la técnica de etnografía del aula bajo los parámetros de las investigaciones cualitativas. Los 
investigadores se involucraron en los procesos de enseñanza y aprendizaje de las diferentes áreas curriculares en la educación básica regular. Asimismo, registraron minuciosamente los diferentes sucesos y pasajes en el ambiente escolar utilizando una grabadora digital, cuadernos de campo y otros medios como grabadora, filmadora y cámara fotográfica.

\section{RESULTADOS Y DISCUSIÓN}

1. Las herramientas conforman y modelan conductas: Los cuadernos de notas

Hace poco tiempo, una maestra intentó ir contra la corriente. Julia inició su clase de comunicación integral dictando un poema y pidiendo a las alumnas de sexto grado de primaria que lo releyeran varias veces. «Por favor, quiero que anoten en los márgenes lo que el poema significa para ustedes», dijo. En el subsiguiente diálogo general, un estudiante tras otro fueron interviniendo, y la clase reconstruyó el poema. «Leamos otro poema», dijo Julia. «En un trozo de papel, escriban sus ideas». Después, los alumnos comentaron el poema, de a dos líneas por vez.

Esto era escribir para aprender, pero algo no estaba bien en la tarea. Lo que estamos sugiriendo es que el contexto de ese hecho de escritura para el aprendizaje lo hacía aparecer como uno más de los tantos ejercicios dirigidos por el docente. «Lo que quiero que hagan...», había dicho Julia: «Escriban sus ideas...» La escritura fue hecha en los márgenes de un texto suelto y en un papelito. Si Julia hubiera iniciado esa unidad de poesía diciendo a los alumnos que iban a leer y escribir sobre poemas a fin de desarrollar y perfeccionar estrategias de interpretación poética, las alumnas podrían haber comprendido que en las lecturas había un propósito acumulativo, que con cada poesía iban a estar mejor preparadas para hacer la tarea interpretativa. Lo habrían sentido más si durante el comentario general Julia hubiera dejado que algunas niñas presentaran su interpretación completa a las compañeras de la clase, para poder después tratar sobre los aciertos y debilidades de su lectura individual. Pero, en cambio, la escritura precedió a una inmediata conversación del conjunto de la clase, en la cual la opinión de cada alumna se amalgamó en una interpretación colectiva. También el hecho de que las niñas hicieran sus anotaciones en papelitos sueltos que no dejan rastro implicó un trato desaprensivo de la escritura de la alumna.

Las herramientas conforman y modelan conductas. Si bien los escritos para el aprendizaje deben ser borradores, llenos de esquemas, listas y fragmentos de ideas, esa escritura debe ser tomada en serio para que los estudiantes la vean como un importante medio de aprendizaje. Si cada una de las alumnas de Julia hubiera elaborado las notas en sus carpetas, la escritura se hubiera parecido menos a un ejercicio para la maestra que a un medio para el desarrollo y registro de las ideas. Quizás con otra perspectiva, Julia podría haber fotocopiado los poemas y dejado espacio en el margen derecho de cada página, permitiendo así que las alumnas tuvieran una carpeta de doble entrada, con los textos de un lado y las notas del otro.

Nosotros no deseamos equiparar la escritura para el currículum con determinada forma de comentarios literarios sino mostrar que tenemos mucho que ganar generando un espacio para la escritura de aprendizaje curricular. Creemos también que los 
maestros se concentran en lo que van a decir y en lo que los alumnos van a decir y a escribir, y prestan menos atención a los mecanismos que estructuran esos hechos de enunciación. Por ejemplo, decidir si un debate será general o en pequeños grupos es mucho más importante para un plan de clase que determinar el contenido de la breve alocución que precederá a ese debate. Y, sin embargo, es posible que atiendan más a las palabras que van a decir que a la forma en que los estudiantes van a trabajar. Del mismo modo, la decisión acerca de si se trabajará en un cuaderno de espiral o en una carpeta de hojas y de si la toma de notas será graduada o no en orden de dificultad creciente es mucho más importante que las palabras que pronuncie la maestra para presentar las actividades de escritura de aprendizaje curricular.

No podemos efectuar una serie de recomendaciones acerca de cómo deberán emplearse los cuadernos de notas: el uso dependerá de su finalidad. Un cuaderno de notas de ciencias probablemente contendrá gráficos y observaciones detallados, con secciones aparte para anotar nuevas preguntas de investigación. Los anotadores de producción escrita contendrán encabezamientos alternativos, diagramas de posibles secuencias, recortes de diarios, títulos, finales, y más listas. Algunas veces de espiral, a veces consistirán en una colección de fichas sobre un tema. La forma seguramente importa, pero no hay una forma única y suprema.

\section{Haciendo preguntas}

Hawkins (1974) señala que pocas personas son problematizadoras y sugiere que eso es particularmente cierto a propósito de nuestra inmersión en el mundo científico y tecnológico que nos rodea; así como destinamos dos minutos a admirar productos tecnológicos -coches, aviones, computadoras, máquinas- y luego pasamos rápidamente del interés al aburrimiento.

Con frecuencia parece que los estudiantes miraran las clases con los ojos suspendidos, esperan que la información venga hasta ellos. El maestro ve esas miradas suspendidas y piensa: «Tengo que ser más estimulante». Las clases se vuelven más animadas, más variadas, hacen piruetas en procura de algún asomo de interés de los alumnos. Desde luego, el maestro está divagando. Se ha quedado con la noción de que se puede «aprender» a los estudiantes. Esto no es solo una incorrección gramatical, es un imposible. Aprender es algo que no se puede hacer por nuestros niños. El aprendizaje exige un acto de iniciativa de su parte. Los maestros solo pueden crear condiciones en las cuales el aprendizaje pueda producirse.

Los cuadernos de notas de aprendizaje pueden contribuir a la creación de esas condiciones, al alentar a los alumnos a hacer preguntas. Nunca se podrá subrayar lo suficiente la importancia del planteo de problemas y de la formulación de preguntas. Por cierto, la habilidad de plantear interrogaciones apropiadas y certeras es una parte crucial de la comprensión, y esto vale tanto para un libro como para una película, una conferencia, un problema de matemáticas o una situación de la vida. Sin embargo, en las escuelas no se enseña a preguntar. Ni siquiera se permite que eso suceda. En la mayoría de las aulas, los estudiantes rara vez formulan preguntas; solo las contestan. En una observación de doce clases, por ejemplo, encontramos solamente nueve casos de 
secuencias de pensamiento iniciadas por los alumnos. El diálogo estaba siempre dirigido por la iniciativa del docente. Formular interrogantes es, sin embargo, la parte más desafiante e importante del pensamiento. Como escribe Wertheimer, «Con frecuencia, en los grandes descubrimientos, lo más importante es dar con determinada pregunta. Encontrar la pregunta productiva es más trascendente que hallar la solución para una pregunta dada».

Los cuadernos de notas dan a los estudiantes un espacio para plantear preguntas. Antes de mostrar una película sobre la guerra del Pacífico, por ejemplo, se puede pedir a los alumnos que anoten las preguntas que tienen sobre la guerra. Después de la película, clase o lectura, ellos pueden volver a su lista de preguntas, marcando aquellas que ya han obtenido respuesta, tachando las que ahora parecen poco significativas y añadiendo otras nuevas que hayan surgido de la actividad. Pero formular preguntas es una cosa, y aprender a hacer preguntas más cuestionadoras y más apropiadas es otra. Los maestros pueden ir un paso más allá y pedir a los alumnos que piensen en sus preguntas: ¿Cuáles fueron las mejores? ¿Qué es lo que hace que una pregunta sea más efectiva que otra? Ellos pueden reunirse en grupos para comparar sus preguntas y seleccionar alguna pregunta importante que luego pueda convertirse en la base de una discusión del aula.

\section{Imaginando hipótesis}

Hace poco tiempo, observamos a una maestra de ciencias que trabajaba con un tercer grado. «QQué es lo que todos los seres vivos necesitan para sobrevivir?, preguntó a las niñas. Ellas se quedaron silenciosas, y luego una mano se levantó. «¿La piel?», sugirió Rosa. «Si uno no tiene piel, lo que el corazón tiene se desparramará todo por ahí». Las compañeras disfrutaron con la imaginación de Rosa, pero la maestra no. Ella suspiró y siguió en busca de una respuesta mejor. Dudosa, Leonor preguntó: «¿Madres?» No, dijo la maestra de ciencias. «Empieza con A», «نंAbuelos?» La experta se dio vuelta y escribió en el pizarrón: Alimento, Aire, Agua.

Lo que la maestra olvidó es que en ciencias, la «adivinación» se llama formulación de hipótesis. En lectura, es predicción. En todos los casos, la presunción -igual que el planteo de preguntas- es una parte crucial del aprendizaje. Pero a menudo, del mismo modo que la maestra de ciencias, los maestros descalifican inadvertidamente las presunciones de los alumnos. «Estás adivinando» ha pasado a ser una expresión negativa, que significa «No hagas conjeturas». Los cuadernos de anotaciones pueden deshacer el daño que se ha hecho.

Antes de que los alumnos vean la película de la Guerra del Pacífico, los maestros pueden pedirles que imaginen y anoten cuáles pueden haber sido las causas del conflicto. Hagan de cuenta que saben cosas que no saben, actúen como si estuvieran seguros de cosas de las que están dudosos, inventen hechos e ideas... y podrán obtener mucho más de cualquier lectura o investigación que (eventualmente) hagan (Elbow, 1981). Esa «versión instantánea» lanzará a los estudiantes a una posición de iniciativa y dominio. Mirarán una película de otra manera porque han invertido energía en el tema. «Imaginé bien», se dirán, o «No pensé en eso». 
A mitad de camino, antes de que todas las causas de la Guerra del Pacífico hayan quedado de manifiesto, los alumnos pueden volver atrás y revisar su versión instantánea a la luz de lo que ya han aprendido. O la película puede ser proyectada toda de una vez, dando tiempo al final para reflexionar sobre la estrategia de suposiciones. Algunos alumnos habrán basado sus versiones instantáneas en un relato recordado, en las palabras de alguna canción o en su conocimiento de otras guerras. Otros no habrán pensado en establecer conexiones, ni en buscar indicios dentro de sus mentes, sino que habrán hecho «adivinaciones».

Los cuadernos de notas se pueden usar de manera semejante para la lectura, y aquí nuevamente habrá tiempo para que los alumnos reflexionen sobre sus hipótesis y sus predicciones. ¿Eran predicciones libres o se basaban en pistas proporcionadas por el autor? ¿Qué indicios daba el texto? La buena adivinación es una técnica, y los estudiantes pueden usar los anotadores para desarrollarla.

\section{Organizando la información}

Una estudiante universitaria trajo un largo borrador sobre la «relectura de literatura» para su disertación. En el trabajo, resumía los estudios que había hecho; le preguntamos: «¿Qué estás tratando de decir acá? Pareció sorprendida por la pregunta y dijo algo sobre informar acerca de los diversos trabajos sobre el tema. Volvimos a preguntarle qué había descubierto en esa búsqueda. No pudo responder a esa pregunta. No se había guiado por sus opiniones sobre el material; simplemente había leído y había informado lo que otros decían. Le sugerimos que volviera a leer sus notas e iniciar una escritura libre, diagramando, organizando, comentando y resumiendo el tema.

Pensemos en esa estudiante, y en otros estudiantes como ella. ¿Por qué muchos alumnos universitarios parecen incapaces de poner en juego su propio pensamiento para acceder a nuevas informaciones? Nosotros pedimos algo realmente simple: queremos que los estudiantes piensen, discutan con su información, vean regularidades en los datos recogidos, construyan ideas a partir de los datos. Parece un pedido sencillo, pero la triste verdad es que no lo es. En las escuelas y aun en algunas universidades, cuando los estudiantes producen informes se les dan los subtítulos para esos informes, que no pocas veces son subtítulos extraídos de las enciclopedias. Los alumnos raramente tienen que descartar, ordenar o resumir información: solo necesitan hacer saber que han leído. Aun cuando contesten preguntas al final de los textos, ellos pueden estar seguros de que si una pregunta dice «¿Cuáles son las tres causas...?», en algún lugar del texto aparecerá la enunciación ordenada de las tres causas. En las escuelas, los estudiantes tienen muy pocas oportunidades de organizar la información por sí mismos, y aunque esto sea una parte crucial de la escritura y del aprendizaje.

Frank Smith (1978) destaca la conexión entre lectura y organización. Habla de un experimento en el que se entregaron a dos grupos de personas cincuenta barajas con figuras. Al primer grupo se le pidió que memorizara lo que había en las barajas, ya que diez minutos más tarde debería repetirlo. A los miembros del segundo grupo, que desconocían esas instrucciones, se les dijo que organizaran sus barajas en categorías. Pasados diez minutos, se preguntó a ambos grupos qué podían recordar del contenido 
de las barajas. El segundo grupo recordó mucho más que el primero. Smith registra este experimento para destacar que aprendemos organizando, construyendo categorías. ¿Cuántas veces los alumnos arman, critican, reconstruyen y defienden sistemas de organización? Los maestros por lo general se quejan de que los estudiantes de todas las edades tienen dificultades para estructurar bien sus composiciones. Nosotros nos preguntamos si se trata de un problema de escritura o de un problema de pensamiento. Los cuadernos de notas pueden usarse para alentar en los niños la construcción de sistemas de organización. Aquí, presentamos algunas maneras en las cuales se puede hacer esto:

Después de escuchar una clase o leer un texto, los alumnos trazarán mapas en los que representen la estructura de la información. Por ejemplo, un mapa de la presente ponencia podría tener este aspecto:

Mapa de organización de información

$\square$ Relato sobre una maestra.

$\prod$ Punto central: Los cuadernos de notas pueden convertir a los alumnos en aprendices activos.

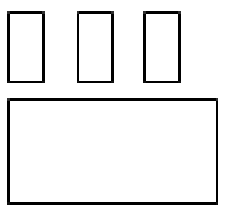

Tres componentes del aprendizaje y cómo los cuadernos de notas pueden crear ocasiones para cada uno.

Resumen y consejos.

Otro mapa de este capítulo podría ser de esta forma:

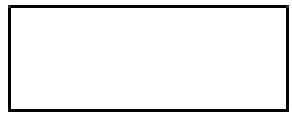

Introducción

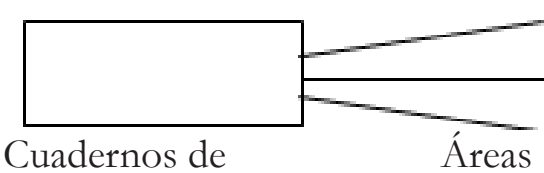

del currículum

Lo importante sería que los alumnos pensaran acerca de la estructura de un texto en vez de producir el mapa «correcto».

Los alumnos pueden anotar detalles sobre cualquier tema -sea la división de números decimales, las nubes o la guerra- y luego pensar en diversas maneras de categorizar la información. Por ejemplo, su información sobre la división de números decimales se puede dividir en estas tres categorías o en otras:

- En qué se parece la división de números decimales a la división de números naturales, y en qué se diferencia.

- Los pasos que se da para resolver un problema de división de números decimales.

- El significado de los términos que se usa en los problemas de división y multiplicación. 
Después de recoger información sobre un tema, se puede inducir a los alumnos a que repiensen y relean la información, categorizándola o buscando una línea argumental o una visión organizativa. Luego, en sus cuadernos de notas, ellos pueden anotar las regularidades observadas.

Para aprender hacemos también muchas otras cosas, y nosotros invitamos a los lectores a encontrar sus propias formas de usar los cuadernos de notas como un espacio para el aprendizaje activo y como una herramienta para generar hábitos de pensamiento. Los maestros pueden querer, por ejemplo, que los alumnos se den cuenta de que el aprendizaje no significa meramente recibir nuevas masas de información. Por el contrario, cada nueva información se debe incorporar en la propia mente. Para realizar este proceso - de asimilación y ubicación- los aprendices deben pensar acerca del nuevo conocimiento en términos del conocimiento preexistente. Los cuadernos de notas pueden ayudarlos a «activar los conocimiento previos», lo cual constituye una vía razonable para empezar cualquier tipo de aprendizaje. Una clase sobre la sábila puede comenzar dando oportunidad a los alumnos de que se pregunten: «¿Qué es lo que yo conozco acerde de para qué es útil la sábila?», y luego, después de adquirir nuevos conocimientos, ellos pueden volver sobre sus pasos y preguntarse: «¿́́mo se adecúa esta nueva información a la que yo ya tenía?», y finalmente: «¿Qué cosas me sorprendieron? ¿Qué fue lo que no se adecuó? ¿Qué hago con esa inadecuación?».

Si el maestro pide a los alumnos que usen cuadernos de notas en el curso de una clase sobre la sábila, es importante que a la semana siguiente, cuando la clase se refiera a otro producto natural, se utilice las anotaciones de modo semejante. Idealmente, se puede pedir a los alumnos que escriban lo que ya saben y que luego integren la nueva información a los esquemas previos no solo en ciencias sino también en matemática o en lectura. Esto es lo contrario de lo que se suele hacer en las escuelas. Los maestros tienden a mantener la variedad, usando el cuaderno de notas de un modo diferente cada día. Pero el problema es que los alumnos nunca tienen la oportunidad de construir y revisar sus estrategias de aprendizaje en una secuencia de días. No pueden elaborar formas de perfeccionar ninguna estrategia en particular porque siempre están pasando de una a otra. Además, si los alumnos realizan nuevas operaciones en cada entrada en el cuaderno de notas, probablemente considerarán que la asimilación de nueva información, o el planteo de preguntas, o la formulación de hipótesis son tareas que se aplican particularmente al tema en cuestión y no hábitos de pensamiento que forman parte del repertorio de todo buen aprendiz. Nuestra aspiración es que los alumnos, en sus cuadernos de notas, externalicen formas de pensamiento que posteriormente internalizarán. Así como las estrategias de revisión se practican primero en forma completa y sistemática y, transformadas ya en rutina se incorporan luego casi sin esfuerzo, las operaciones de aprendizaje pueden internalizarse. Nuestro propósito al usar cuadernos de notas no es solo incitar a los alumnos a pensar, sino también enseñarles hábitos de pensamiento.

Es importante recordar que, al hacer esas operaciones con la escritura, los aprendices adquieren una mayor conciencia y un mayor dominio de su pensamiento. Nosotros empezamos esta ponencia diciendo que «la escritura nos da conciencia de nuestros pensamientos y nos permite organizarlos, nos obliga a detenernos sobre las ideas el 
tiempo necesario para examinarlas, nos hace pensar sobre nuestros pensamientos». Releer la propia escritura, volver sobre las huellas del propio pensamiento, es indispensable. Sartre dejó de escribir cuando perdió la vista porque necesitaba visualizar su pensamiento para manipularlo y desarrollarlo, para componer su significado (Sartre, 1975).

Debemos incluir también un llamado de atención. Tendemos a pedir a los alumnos que relean sus anotaciones para corregirlas y completarlas, particularmente después que han recibido nuevas informaciones procedentes de una clase, de una película o de un texto. Si los estudiantes anotan sus preguntas antes de ver un filme, instintivamente querríamos decirles: «Como tarea para esta tarde, contesten sus preguntas». No hay nada de perjudicial en eso (excepto que enseñemos a los alumnos a plantearse preguntas de respuesta corta), pero pensamos que sería mucho más valioso pedir a los alumnos, como lo sugerí antes, que relean y reconsideren sus preguntas y sus estrategias de pensamiento a la luz de la nueva información. ¿Cuáles fueron sus preguntas más efectivas? ¿Qué es una buena pregunta? Donaldson escribe que «las criaturas humanas pueden aprender a ser conscientes de los poderes de sus propias mentes y decidir cuándo y cómo los usarán. Sin embargo, no pueden hacer eso sin nuestra ayuda». Cuando los niños toman conciencia de sus estrategias de pensamiento, se pueden convertir en pensadores más deliberados. En casa, cuando un chico se apresta cómodamente a leer una novela, puede decirse «voy a imaginar de qué se trata» y luego proceder a confirmar o desmentir esa presunción. En la biblioteca, mientras el niño examina materiales, puede decirse «Veamos cómo encaja todo esto», y comenzar a organizar la información en categorías mayores y menores.

\section{CONCLUSIONES}

En el proceso de adquisición y desarrollo de la escritura crítica y reflexiva, el uso de los cuadernos de notas no solo incita a los alumnos a pensar, sino también a enseñarles hábitos de pensamiento. Es importante recordar que, al hacer esas operaciones con la escritura, los aprendices adquieren una mayor conciencia y un mayor dominio de su pensamiento.

La escritura nos da conciencia de nuestros pensamientos y nos permite organizarlos, nos obliga a detenernos sobre las ideas el tiempo necesario para examinarlas, nos hace pensar sobre nuestros pensamientos.

Finalmente, cuando los niños toman conciencia de sus estrategias de pensamiento, se pueden convertir en pensadores más deliberados.

\section{REFERENCIAS BIBLIOGRÁFICAS}

Donaldson, M. 1978. Children's Minds. New York: W. W. Norton and Company.

Elbow, Meter. 1981. Writing with power: Techniques for mastering the writing process. New York: Oxford University.

Hawkins, David. 1974. The informed vision. New York: Agathon Press. 
|| Silvia Valdivia y Juan López

Sartre, Jean-Paul. 1975. Sartre at seventy: An interview. With Michael Contat. New York: Review of books.

Smith, Frank. 1983. Reading like a writer. Language arts.

Fecha de recepción: 02 de febrero de 2015

Fecha de aceptación: 09 de mayo de 2015

\section{Correspondencia}

Silvia Valdivia Yábar

siveroval@terra.com.pe 